\title{
Case Report \\ Monomicrobial necrotizing fasciitis in a white male caused by hypermucoviscous Klebsiella pneumoniae
}

\author{
Gudjon L. Gunnarsson, ${ }^{1}$ Pernille B. Brandt, ${ }^{2}$ Dorte Gad, ${ }^{1}$ Carsten Struve ${ }^{3}$ \\ and Ulrik S. Justesen ${ }^{2}$ \\ ${ }^{1}$ Department of Plastic Surgery, Odense University Hospital, Odense, Denmark \\ ${ }^{2}$ Department of Clinical Microbiology, Odense University Hospital, Odense, Denmark \\ ${ }^{3}$ Department of Bacteriology, Mycology and Parasitology, Statens Serum Institut, Copenhagen, \\ Denmark
}

We report a case of monomicrobial necrotizing fasciitis caused by hypermucoviscous Klebsiella pneumoniae in an immunocompromised white male after travel to China. The K. pneumoniae isolate belonged to the $\mathrm{K} 2$ serotype, and carried the virulence factors $\mathrm{RmpA}$ and aerobactin. To the best of our knowledge this is the first report of necrotizing fasciitis caused by

Received 16 April 2009 hypermucoviscous $K$. pneumoniae resembling the highly virulent $K$. pneumoniae isolates associated with liver abscess syndrome in Asia.

\section{Introduction}

Klebsiella pneumoniae is recognized as an important opportunistic pathogen primarily causing urinary tract infections and bacteraemia. Recently a distinctive syndrome of community-acquired primary liver abscess associated with metastatic infections has emerged, especially in Taiwan and other Asian countries (Fung et al., 2002; Ko et al., 2002; Wang et al., 1998; Yu et al., 2007). These infections occur with high incidence in otherwise healthy individuals and are characteristically caused by highly virulent $K$. pneumoniae isolates belonging to the capsular serotype $\mathrm{K} 1$ or $\mathrm{K} 2$, expressing a distinct hypermucoviscous phenotype. Two plasmid-encoded virulence factors, RmpA (a regulator of the mucoid phenotype), mediating increased protection against the host immune system by upregulating capsule synthesis, and the iron siderophore aerobactin, enabling the bacteria to obtain iron during infection of the host, are significantly correlated with the high virulence of these invasive strains (Yu et al., 2007, 2008). We report a Danish case of necrotizing fasciitis caused by a hypermucoviscous $K$. pneumoniae isolate of capsule serotype $\mathrm{K} 2$, positive for RmpA and aerobactin.

\section{Case report}

A 66-year-old white male sought medical attention because of a 2 week history of watery diarrhoea and a 2 day history of a progressive skin infection of his left forefoot. He had just returned from a 2 week holiday in Shanghai, China. The patient neither smoked nor had any history of alcohol abuse. He was slightly overweight and received treatment for several medical conditions, including a recently diagnosed type 2 diabetes $(500 \mathrm{mg}$ metformin once a day), ankylosing spondylitis (25 mg methotrexate weekly, $2 \mathrm{~g}$ sulfasalazine and $17.5 \mathrm{mg}$ prednisolone once a day), mild hypertension (5 $\mathrm{mg}$ amlodipine once a day) and hypercholesterolaemia (40 mg simvastatin once a day). The patient's podiatry hygiene was optimal and he never suffered from leg ulcers, calluses or corns. He denied any previous wounds of his legs, although he suggested that he might have been bitten by an insect in the ankle without knowing it. The patient's diabetes was mild and recently diagnosed, and his blood sugar level was monitored as stable within the normal range with no hyperglycaemic episodes occurring during his hospital stay.

The patient had consulted his general practitioner a few days earlier because of the diarrhoea and skin infection, and treatment with $800 \mathrm{mg}$ phenoxymethylpenicillin three times a day was initiated, without any effect. On admittance he was tired and showed clinical signs of dehydration but had no fever. He had cellulitis of the entire dorsal forefoot extending circularly halfway up to his knee. During the first hours of observation he suddenly developed signs of sepsis, and in a very short time the foot and lower leg became increasingly painful with the appearance of bullae, and eventually the skin infection evolved to a fulminant necrotizing cellulitis.

Treatment with intravenous $2 \mathrm{~g}$ meropenem, $500 \mathrm{mg}$ metronidazole and $600 \mathrm{mg}$ clindamycin three times a day was initiated. Clindamycin was discontinued the following day as Gram-negative bacilli were recovered in blood cultures. Metronidazole was discontinued after 7 days. 
When the patient developed bullae, immunoglobulins were given in case this was a sign of the development of a toxic shock syndrome ( $30 \mathrm{~g}$ gamma globulin once a day for 3 days). Intravenous immunoglobulins have been associated with an improved outcome in small studies of streptococcal toxic shock syndrome, which can accompany necrotizing fasciitis caused by group A streptococcus (Darenberg et al., 2003). However, there are no studies to support the use of immunoglobulins to treat necrotizing fasciitis in general.

An operation was performed immediately and the infected skin, subcutis and underlying necrotic fascia were removed. The patient's wounds were treated with a negative pressure device and 12 hours later the leg was re-explored without any signs of further tissue necrosis being found. The patient was clinically stable with signs of infection already subsiding. Five days following the initial operation, skin grafts were applied to the wounds. The patient made a slow recovery due to delayed wound healing complicated by secondary infection with Pseudomonas aeruginosa. The patient's diarrhoea subsided without treatment. Antibiotic treatment with meropenem was continued due to signs of right-sided lobar pneumonia considered to be secondary to aspiration. Liver function tests were all normal and there were no further symptoms indicating other organ involvement. After 5 days of meropenem treatment the patient developed severe oral candidiasis that was treated with $100 \mathrm{mg}$ fluconazole once a day. He was discharged after 45 days of hospitalization.

Cultures of blood and wound debris all grew $K$. pneumoniae susceptible to ceftazidime, ceftriaxone, meropenem, ciprofloxacin, gentamicin, tetracycline and polymyxin. All cultures of faeces were negative for $K$. pneumoniae. The K. pneumoniae isolates from the wound debris and blood were determined to express the hypermucoviscous phenotype defined as formation of a string $(>5 \mathrm{~mm}$ ) when a loop is passed through a colony (Fang et al., 2004). Capsular serotyping carried out at the International Escherichia coli and Klebsiella Reference Centre (World Health Organization), Statens Serum Institut, Denmark, revealed that the isolate belonged to the K2 serotype. Furthermore, the isolate carried the virulence factors RmpA and aerobactin as revealed by PCR using specific primers. Thus the isolate exhibited similar characteristics to the highly virulent $K$. pneumoniae isolates associated with liver abscess syndrome in Asia.

\section{Discussion}

Historically, K. pneumoniae is a rare cause of monomicrobial necrotizing fasciitis, with the earliest case being reported in 1996 (Wong et al., 2004). Intriguingly, the majority of reported cases have been from Asia and the incidence of necrotizing fasciitis caused by $K$. pneumoniae seems to be rising. Thus, $K$. pneumoniae was recently found to be the most commonly identified bacterium in a study of necrotizing fasciitis in Taiwan (Liu et al., 2005). Only two cases have been reported from Europe and the USA: a newborn child from Turkey with a gangrene of the perineum (Özkan et al., 1997) and a Cambodian male hospitalized with monomicrobial necrotizing fasciitis and septic arthritis after a 6 months stay in the USA (Kohler et al., 2007). It could be speculated that the higher incidence in Asia of necrotizing fasciitis caused by K. pneumoniae might be related to the high prevalence of virulent hypermucoviscous $K$. pneumoniae strains in this region. Indeed, necrotizing fasciitis has been reported in relation to the community-acquired $K$. pneumoniae liver abscess syndrome (Dylewski \& Dylewski, 1998; Hu et al., 1999; Wong et al., 2004). The vast majority of the highly virulent hypermucoviscous $\mathrm{K}$. pneumoniae isolates belong to the $\mathrm{K} 1$ or $\mathrm{K} 2$ serotype, and the virulence factors RmpA and aerobactin have been shown to correlate with the enhanced virulence of these isolates compared to non-hypermucoviscous $\mathrm{K} 1$ and $\mathrm{K} 2$ isolates (Yu et al., 2007, 2008). To the best of our knowledge, capsule serotyping of K. pneumoniae isolates causing necrotizing fasciitis has only been performed in two cases, and in both of these the infection was caused by isolates of the $\mathrm{K} 1$ serotype (Hu et al., 1999; Kohler et al., 2007). However, no previous studies have investigated necrotizing fasciitis isolates for specific virulence factors including the hypermucoviscous phenotype, RmpA and aerobactin. It is likely that our patient was colonized by the $K$. pneumoniae isolate during his recent stay in China, although it cannot be excluded he was already colonized by the K. pneumonia strain in Denmark. Indeed cases of community-acquired liver abscess caused by hypermucoviscous $K$. pneumoniae in patients with no history of travelling to Asia have been reported from Europe and the USA, demonstrating the emergence of these strains outside of Asia (Frazee et al., 2009; Gomez et al., 2007; Karama et al., 2007).

We emphasize the importance of early surgical debridement to reduce bacterial and toxin load in patients with aggressive necrotizing cellulitis, which can evolve to necrotizing fasciitis. Skin signs are usually late in necrotizing fasciitis and it is more common for a patient with necrotizing fasciitis caused by K. pneumoniae to have associated organ abscesses signifying that the infection is probably haematogenously spread (Ho et al., 2000; Yu et al., 2007). The fact that our patient had no such foci supports the belief that this case was initially a skin infection, which is less common. The prognosis was subsequently better as the disease is localized to the limb involved and surgical delay is minimized. There are several studies showing higher incidence of opportunistic infections in patients with rheumatological diseases, and a wide array of case reports relate the use of corticosteroids for mild and serious infections. Methotrexate is known to cause alterations in humoral and cellular immunity, but data suggesting it causes immunosuppression when used at low therapeutic doses are debatable (McLean-Tooke et al., 2009). There is, however, no doubt that all individual factors synergistically increase the risk of infection. 
To the best of our knowledge this is the first reported case of monomicrobial necrotizing fasciitis caused by K. pneumoniae in Western Europe. Furthermore, it is believed to be the first case where it has been established that the infection was caused by a hypermucoviscous K. pneumoniae isolate of the K2 serotype carrying the virulence factors RmpA and aerobactin, thereby resembling the highly virulent $K$. pneumoniae isolates associated with liver abscess syndrome in Asia. The rising incidence of severe infections caused by highly virulent $K$. pneumoniae isolates is worrisome and calls for increased international surveillance, including virulence factor assessment, typing and registration of isolates to monitor the geographical spreading of these infections. With this case report we wish to increase the awareness of other serious infections apart from liver abscess caused by hypermucoviscous $K$. pneumoniae, such as necrotizing fasciitis. Due to an ageing population, advances in medical care and treatment modalities, along with more frequent travelling between the continents, infections caused by these aggressive bacteria might become more frequent in our practice in the future.

\section{References}

Darenberg, J., Ihendyane, N., Sjölin, J., Aufwerber, E., Haidl, S., Follin, P., Andersson, J., Norrby-Teglund, A. \& the Streptlg Study Group (2003). Intravenous immunoglobulin $G$ therapy in streptococcal toxic shock syndrome: a European randomized, doubleblind, placebo-controlled trial. Clin Infect Dis 37, 333-340.

Dylewski, J. S. \& Dylewski, I. (1998). Necrotizing fasciitis with Klebsiella liver abscess. Clin Infect Dis 27, 1561-1562.

Fang, C. T., Chuang, Y. P., Shun, C. T., Chang, S. C. \& Wag, J. T. (2004). A novel virulence gene in Klebsiella pneumoniae strains causing primary liver abscess and septic metastatic complications. J Exp Med 199, 697-705.

Frazee, B. W., Hansen, S. \& Lambert, L. (2009). Invasive infection with hypermucoviscous Klebsiella pneumoniae: multiple cases presenting to a single emergency department in the United States. Ann Emerg Med 53, 639-642.

Fung, C. P., Chang, F. Y., Lee, S. C., Hu, B. S., Kuo, B. I., Liu, C. Y., Ho, M. \& Siu, L. K. (2002). A global emerging disease of Klebsiella pneumoniae liver abscess: is serotype $\mathrm{K} 1$ an important factor for complicated endophthalmitis? Gut 50, 420-424.
Gomez, C., Broseta, A., Otero, J. R. \& Chaves, F. (2007). Primary pyogenic liver abscess caused by $m a g A^{+}$Klebsiella pneumoniae in Spain. Clin Microbiol Newsl 29, 100-102.

Ho, P. L., Tang, W. M. \& Yuen, K. Y. (2000). Klebsiella pneumoniae necrotizing fasciitis associated with diabetes and liver cirrhosis. Clin Infect Dis 30, 989-990.

Hu, B. S., Lau, Y. J., Shi, Z. Y. \& Lin, Y. H. (1999). Necrotizing fasciitis associated with Klebsiella pneumoniae liver abscess. Clin Infect Dis 29, 1360-1361.

Karama, E. M., Willermain, F., Janssens, X., Claus, M., Van den Wijngaert, S., Wang, J. T., Verougstraete, C. \& Caspers, L. (2007). Endogenous endophthalmitis complicating Klebsiella pneumoniae liver abscess in Europe: case report. Int Ophthalmol 28, 111-113.

Ko, W.-C., Paterson, D. L., Sagnimeni, A. J., Hansen, D. S., Von Gottberg, A., Mohapatra, S., Casellas, J. M., Goossens, H., Mulazimoglu, L. \& other authors (2002). Community-acquired Klebsiella pneumoniae bacteremia: global differences in clinical patterns. Emerg Infect Dis 8, 160-166.

Kohler, J. E., Hutchens, M. P., Sadow, P. M., Modi, B. P., Tavakkolizadeh, A. \& Gates, J. D. (2007). Klebsiella pneumoniae necrotizing fasciitis and septic arthritis: an appearance in the western hemisphere. Surg Infect (Larchmt) 8, 227-232.

Liu, Y. M., Chi, C. Y., Ho, M. W., Chen, C. M., Liao, W. C., Ho, C. M., Lin, P. C. \& Wang, J. H. (2005). Microbiology and factors affecting morality in necrotizing fasciitis. J Microbiol Immunol Infect 38, 430-435.

McLean-Tooke, A., Aldridge, C., Waugh, S., Spickett, G. P. \& Kay, L. (2009). Methotrexate, rheumatoid arthritis and infection risk - what is the evidence? Rheumatology 48, 867-871.

Özkan, H., Kumtepe, S., Turan, A., Funda, Corapçioglu \& Özkan, F. (1997). Perianal necrotizing fasciitis in a neonate. Indian J Pediatr 64, $116-118$.

Wang, J.-H., Liu, Y.-C., Lee, S. S.-J., Yen, M.-Y., Chen, Y.-S., Wang, J.-H., Wann, S.-R. \& Lin, H.-H. (1998). Primary liver abscess due to Klebsiella pneumoniae in Taiwan. Clin Infect Dis 26, 1434-1438.

Wong, C. H., Kurup, A., Wang, Y. S., Heng, K. S. \& Tan, K. C. (2004). Four cases of necrotizing fasciitis caused by Klebsiella species. Eur $\mathrm{J}$ Clin Microbiol Infect Dis 23, 403-407.

Yu, V. L., Hansen, D. S., Ko, W. C., Sagnimeni, A., Klugman, K. P., von Gottberg, A., Goossens, H., Wagener, M. M., Benedi, V. J. \& the International Klebseilla Study Group (2007). Virulence characteristics of Klebsiella and clinical manifestations of K. pneumoniae bloodstream infections. Emerg Infect Dis 13, 986-993.

Yu, W. L., Ko, W. C., Cheng, K. C., Lee, C. C., Lai, C. C. \& Chuang, Y. C. (2008). Comparison of prevalence of virulence factors for Klebsiella pneumoniae liver abscesses between isolates with capsular K1/K2 and non-K1/K2 serotypes. Diagn Microbiol Infect Dis 62, 1-6. 